\title{
Computing Cell-Based Decompositions Dynamically for Planning Motions of Tethered Robots
}

\author{
Reza H. Teshnizi and Dylan A. Shell
}

\begin{abstract}
Recently researchers have approached the problem of motion planning with topological constraints. In such problems, the inputs to the planner are source and destination points and the output is expected to be a valid path that respects the topological constraints. A concrete example of such problems - and the topic of this paper-is planning for a robot which is connected with a cable of limited length to a fixed point in the operation space. This paper presents a planning method for such problems by examining how the configuration space manifold can be represented efficiently. We introduce a convenient method for generating either parts or the complete atlas for the manifold based on special "cable events". Generating parts of the configuration space on-the-fly enables improvements over the state of the art: (a) we decompose the environment into cells as needed rather than an off-line global discretization, obtaining competitive time and space complexity for our planner, (b) we are able to exploit topological structure to represent robot-cable configurations concisely, (c) we generalize the representation in order to examine cable-to-cable contacts, which have been widely ignored in the literature until now. Our results show the efficiency of the method and indicate further promise for procedures that represent manifolds via an amalgamation of implicit discrete topological structure and explicit Euclidean cells.
\end{abstract}

\section{INTRODUCTION}

The classic problem of motion planning can be defined as moving an object in a space while avoiding obstacles [1]. Many practical scenarios require more complicated variations of the problem. Consider a robot which uses a cable as a source of power or communication. Examples include high power robots (e.g., street cleaning machines) that use cable as a source of power and underwater or underground robots that use a cable for transferring data. Presence of the cable imposes two important constraints on the robot's motion: the cable length which limits the radius of the movement (Fig. 1a), and the topological constraints imposed by the cable and obstacles in the environment (Fig. 1b).

Recent research has focused on different aspects and variations of this problem [2]-[5]. The present work examines the structure of the configuration space induced by a robot tethered by a taut (possibly retracting) cable and proposes to represent the manifold of configurations by a special atlas [6]. In so doing, the topological regularity captured as a graph, is naturally separated from the continuous aspects captured as charts. The graph provides an understanding of the complexity induced by the cable and nodes within the graph provide sufficient topological context for points in $\mathbb{R}^{2}$

R. H. Teshnizi and D. A. Shell are with the Distributed AI and Robotics Lab, Dept. of Computer Science and Engineering, Texas A\&M University, College Station, TX 77843, USA. Emails: $\{r$. teshnizi, dshell\}ecse.tamu.edu

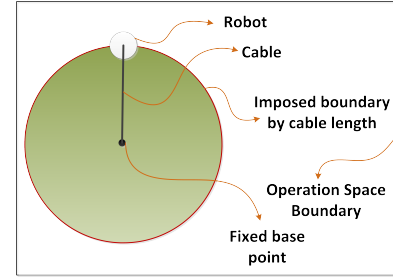

(a) Length Constraint

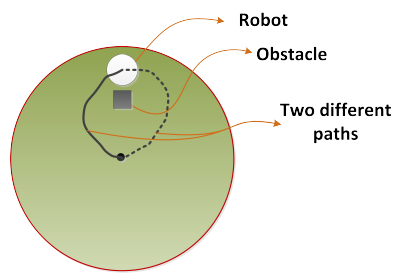

(b) Topological Constraint
Fig. 1: The limits caused by fixing the robot to a fixed point

to represent configurations. The planning method we present is quite straight forward using this representation.

This method creates a set of locally continuous charts based on the set of cable events. Initially, we examine events that occur when a cable touches or wraps around an obstacle, and we show that they are intimately connected with a subgraph of the visibility graph of the environment. We will show that for a tethered robot this subgraph is in fact a tree and thus the topological structure of the atlas representing the configuration space forms a tree structure. This structure provides context that allows path planning to proceed by moving locally (either up to parent charts, or downward to children charts) in the configuration space.

Next, we extend the set of cable events to consider cableto-cable interactions, by considering two different ways to cross the cable: the robot can move over or under the cable $^{1}$. The method-we believe, uniquely-generalizes to this case despite the fact that the involved topology becomes rather more complex: events no longer occur at a countable set of locations, the configuration space's structure is no longer separated easily. Nevertheless, because only parts of the configuration manifold need be generated and the path planning operations proceed exploring locally, the approach succeeds. We believe that this underscores the strength of dynamically computing relevant parts of the atlas on-the-fly and treating the configuration space as if it were a just-intime computed data structure.

\section{RELATED WORK AND CONTRIBUTIONS}

Much prior work on motion planning for tethered robots has the common underlying idea of creating a graph approximation of the configuration space and using an efficient method to search through the space of possible paths to choose the optimal one among those in the homotopy class.

The work of Hert and Lumelsky [7] first considered the problem of ordering the motions of multiple tethered

\footnotetext{
${ }^{1}$ We do assume in this case, that the cable has infinite friction in contacting itself.
} 
robots by sorting a graph representing their paths. While influential, the paper never addressed finding those orderings. The work of Grigoriev and Slissenko [8] and Narayanan, Vernaza, Likhachev, and LaValle [9] addressed the problem by representing paths in a given homotopy class by defining an alphabet used to describe the event of crossing a ray. Determining whether two paths are in the same homotopy class then requires comparison of their related strings. The latter paper also addresses homologically equivalence. That research group also explored the topic of winding constraints for motion planning [4]. A radically different approach is taken by Bhattaharya, Kumar, and Likhachev [2], who provide an alternative way of defining homotopy classes of paths in 2D based on the Cauchy Integral Theorem.

Igarashi and Stilman [3] designed an algorithm for creating a graph of the configuration space manifolds based on cable length constraint. Their idea of representing the configuration space by multiple overlapping manifolds influenced the present work. Recent work has also examined problems beyond path planning in tethered robots [5].

Based on the body of prior work, we have identified several distinguishing contributions of the current paper. Firstly, the approach we propose avoids discretizing the configuration space, instead using a subset of the visibility graph to induce a natural cell decomposition of the space. We need to clarify what we mean by decomposition versus discretization: decomposition divides a space into subspaces which are locally continuous whose union is equal to the original space, whereas a discretization's faithfulness to the original space depends on its resolution. Secondly, it connects motions of the robot to discrete events that occur with the cable, each representing qualitative changes in the robot and cable configuration. The idea of identifying events of this form allows the method to be generalized so as to model cable-cable interactions appropriately. Thirdly, we avoid offline creation of the entire configuration space, keeping data structures that allow for dynamic generation of necessary parts of the space. We represent topological context and local metric information, where planning in the local chart is trivial, and new charts are only created on-the-fly as needed. And finally, the visibility graph has been previously used in motion planning for non-tethered robots [10], [11], but our construction of an atlas of charts leads to new insight with regards to the cable's dependency on the environment topology and its connection with the visibility graph.

\section{The Preliminaries}

This section provides the fundamental definitions used throughout the paper. We consider the problem of planning for a non-oriented robot situated in a planar environment with a cable tethered to a fixed point. It is assumed that the cable of maximum length $l$ is always taut (e.g., through the use of a retracting or spooling mechanism). And also that the obstacles are known and polygonal.

Figs. 2a-2c illustrate aspects of the problem. In an obstacle free environment, motion planning for a tethered robot is identical to an untethered robot with circular boundary of

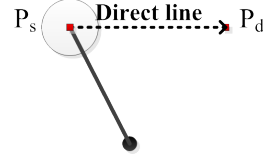

(a) No Obstacles

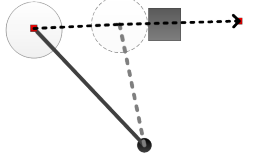

(b) Obstacle blocking (c) Cable-obstacle conthe direct line

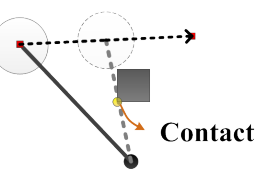

tact
Fig. 2: Different scenarios of a tethered robot with respect to the presence of obstacles.

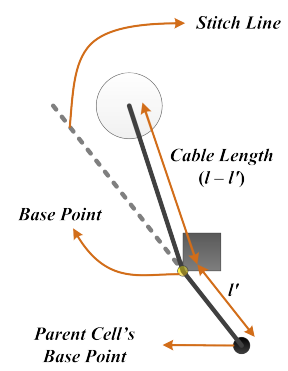

Fig. 3: The defining elements of a visibility cell.

radius $l$. A path from $p_{s}$ to $p_{d}$ is a straight line segment [12]. Let $a \rightarrow b$ denote the line segment connecting point $a$ to $b$. In moving from $p_{s} \rightarrow p_{d}$, an obstacle may affect the robots motion directly (Fig. 2b) or indirectly via a cable-obstacle contact which will bend the cable. Fig. 2c. In the latter case, the radius of movement of the robot after that bend is affected.

\section{A. Events}

Consider the fixed base point of the cable. Due to the limited length of the cable, the distance between the robot and this point is never greater than $l$. Therefore, the accessible area for the robot will be a circle with radius $l$ centered at that point (Fig. 1a). Interestingly, this is true for any contact point as well. If the robot consumes $l^{\prime}$ of its cable length to reach the contact point, since the cable is always taut, its distance from the contact point can never become greater than $l-l^{\prime}$ unless it untangles itself (Fig. 3). This is the basis for cell decomposition of the configuration space.

The contact points also define a homotopy class of trajectories that the robot can follow in order to untangle the cable and get back to the initial configuration (i.e., the cable is completely retracted and the robot is at the origin of the cable). Incidents that change the homotopy class of returning trajectory and/or the boundaries of accessible area for the tethered robot are referred to as events.

Definition 1. In the context of the cabled robot, there are two kinds of events:

1) Cable to obstacle contact: wrapping event

2) Cable to cable contact: cable crossing event

In the following subsections, we are not concerned about cable-cable interactions. We return to cable to cable interactions in Section V where the method is extended.

\section{B. Visibility Cells}

A wrapping event will only occur when the cable touches an apex of one of the polygonal obstacles. These apexes 
are the same as the vertices of the visibility graph [13]. We can construct a connected component of the free space of the environment by partitioning the planar map into a semialgebraic set $P_{o b s}$ consisting of all the obstacles and the free space $P_{\text {free }}$ that is the complement of the set $P_{o b s}$ [8].

Since planning the motion for each cell of a decomposed configuration space is straight forward, we are going to define these cells in a way that the union of them will cover the configuration space. That is, each cell should contain all the points so that no movement of the tethered robot from any point in the cell to any other point in the same cell causes an event. Therefore we can reach the following definition for the cells of the configuration space in our context.

Definition 2. A visibility cell is a chart, $(U, \varphi)$, where $U \subseteq P_{\text {free }}$ and homeomorphism $\varphi$ is $\varphi(x, y)=(x, y)$, and the collision free path between any two points in $U$ is a straight line segment inside $U$ connecting the two points.

We identify cells uniquely by the following fields (Fig. 3):

- Base Point: as discussed in Section III-A, each wrapping point is used as a base point.

- Cable Length: determines the maximum distance between the robot and the base point of a chart.

- Parent Cell: is the cell describing the robot and its cable configuration directly before occurrence of the event. This information is crucial when the planner is searching for paths and/or untangling the cable.

- Stitch Line: this is the line where one chart is connected to another and can be considered as an interface between them. Formally this line is the domain for transition map between the two charts. Once the robot have crossed the stitch line, a contact is made/released and thus the robot will be transfered from a chart (cell) to the other.

Fig. 4 illustrates a 3D model of how the visibility cells are connected together.

\section{The Maximum Ray Length Visibility Atlas}

Since each cell is basically a chart, we next define an atlas as a model of the decomposition.

Definition 3 (informal). A Maximum Ray Length Visibility Atlas (MRLVA) denoted by $A_{l}$ is an atlas which contains visibility cells whose stitch lines are edges of the visibility graph of the environment. Each MRLVA has a graph associated with it characterizing its topological structure, which we call Maximum Ray Length Visibility Atlas Graph (MRLVAG) denoted by $G_{A_{l}}$ (see Fig. 5).

A detailed procedure for creating a MRLVA and MRLVAG is provided in the technical report [14].

It is important to note that number of child charts is always less than or equal to the actual visibility graph's vertices.

Lemma 1. In an environment with polygonal obstacles a locally shortest path has a canonical form: in $P_{\text {free, }}$ it is a straight line segment connecting the two end points. If one of the end points meets an obstacle it is locally supporting to $P_{\text {obs }}$ at the contact point [12].

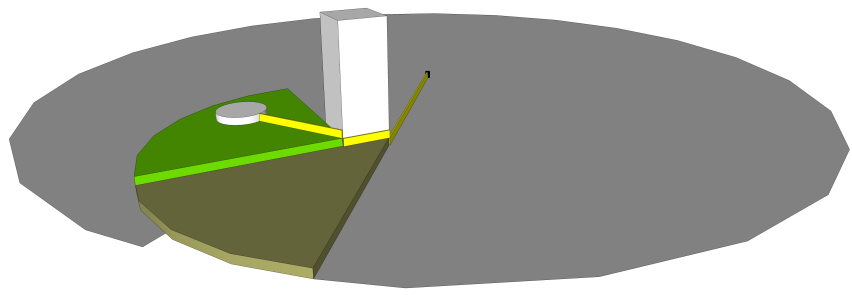

Fig. 4: A 3D model of the visibility cells and the way they are connected together. Each cell is in a different color. The robot is white and the cable is colored yellow.
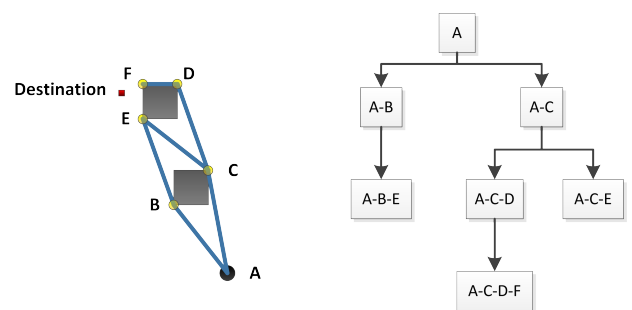

Fig. 5: An example of a MRLVAG. The environment producing this MRLVAG is given on the left.

Theorem 1. The MRLVA $A_{l}$ generated on $P_{\text {free }}$ describes exactly the space of all the possible configurations of a robot (non-oriented) and its taut cable with maximum length $l$ in $P_{\text {free. }}$

Proof. The tautness of the cable implies each segment of it is a locally shortest path. So by Lemma 1 it is always in form of a line segment in $P_{\text {free }}$ and these line segments connect apexes of the obstacles which are a subset of edges of visibility graph, hence constituting all the configurations of the taut cable, except the final segment connecting the robot to the last apex. This last piece of information is provided by the position of the robot represented as a point in a visibility cell (that is a chart). Therefore, by connecting this point to the last apex we will have the all the robot and its taut tether's configurations.

Corollary 1. Given a MRLVA, by having a path from the root to a chart $\left(U_{i}, \varphi_{i}\right) \in A_{l}$ and a point $p \in U_{i}$ can identify a unique configuration of a non-oriented robot and its taut tether in $P_{\text {free }}$.

Proof. Follows from Theorem 1 and Lemma 1.

Theorem 2. The MRLVAG $G_{A_{l}}$ generated on $P_{\text {free, }}$ is a tree.

Proof. Suppose, to the contrary, that $G_{A_{l}}$ has a loop. This implies two distinct sequences of cells and their transition maps to reach same cell. Each sequence implies an ordering of apexes, that is two distinct configurations of the cable for reaching the same cell. This contradicts Corollary 1.

\section{The Basic Planning Algorithm}

Algorithm 1 shows the pseudo code for finding a path from source point $p_{s}$ to destination point $p_{d}$. This algorithm 
is greedy since it values the direct line connecting the source point to the destination point more than other paths.

In order to initiate the algorithm the robot calls FindPath for current chart given the source and destination points. This function first considers $p_{s} \rightarrow p_{d}$ as it is always the locally shortest path [8]. If it cannot move directly toward $p_{d}$, it then checks whether a path can be obtained either from its children or its parent. If there is no child or the cable is not long enough, search in this branch of atlas tree will be terminated. Otherwise, the planner searches through all the children and stores the shortest path found in them. Next it ensures no shorter path from the parent chart to the destination point exists, otherwise the final path will go through the parent chart instead.

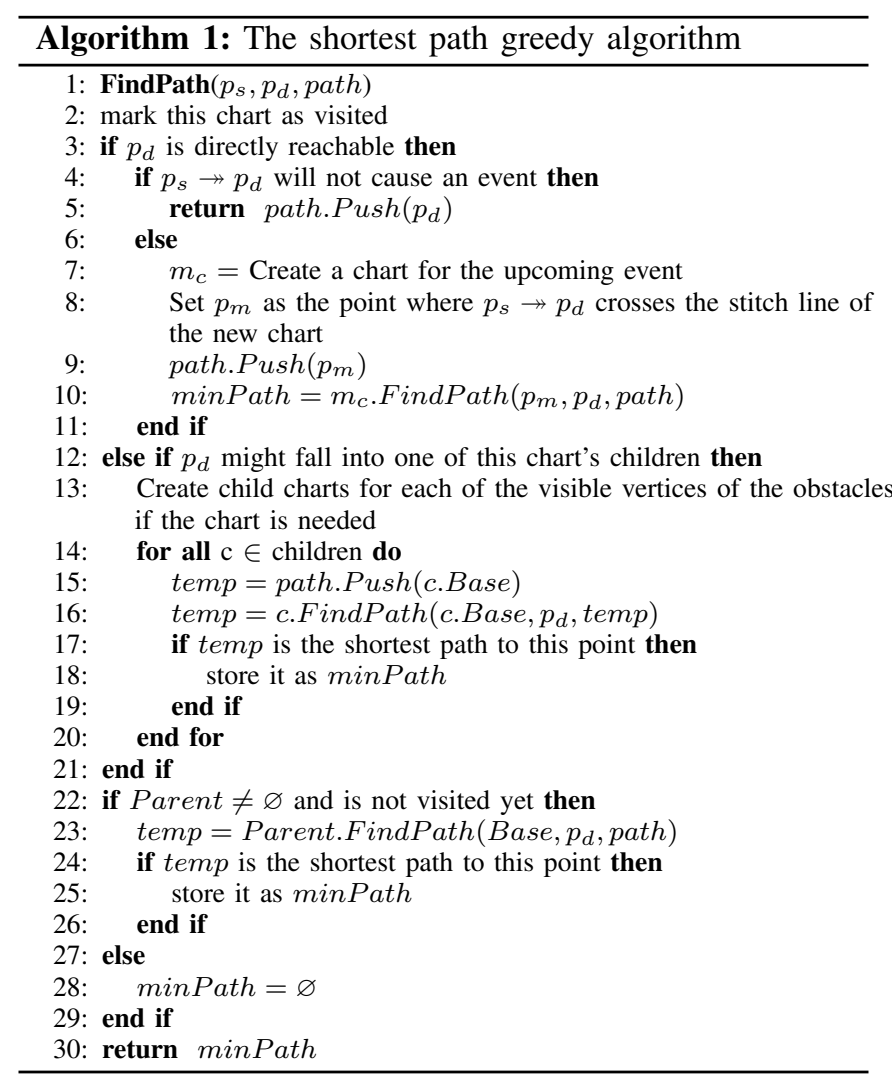

\section{Handling CABle-Cable Interaction}

This section discusses how to handle a robot crossing its own cable and the importance of these events.

\section{A. Cable-Cable Interaction Events}

Previous work generally opts to ignore instances in which a robot's path crosses the cable. An exception is [3] wherein the authors deliberately plan movements to avoid such paths. The main reason of ignoring the cable-to-cable contacts is that it is either impossible to model the cable contact points, or it is computationally inefficient to do so (e.g., discretizing the cable.) In contrast, we show that the MRLVAG can be extended to consider events of this type so that the cable configuration is stored dynamically.

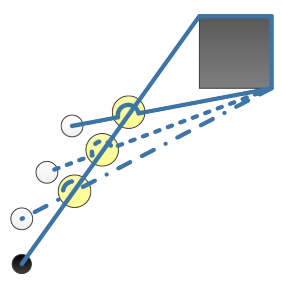

(a)

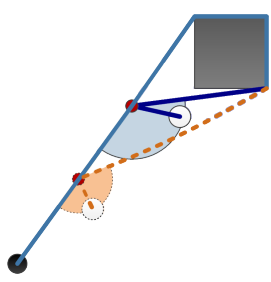

(b)
Fig. 6: The complexities of the cable-to-cable contacts: (a) Uncountable contact points and (b) changing movement radius.

Mainly, modeling this situation is complicated for two reasons: (a) the cable can make contact to another segment of the cable in uncountable number of points, (b) the radius of feasible subsequent movements depends on the location of the contact point (see Fig. 6).

When encountering the cable, the robot may either go over the cable or under it. For these cases, the algorithm is modified by checking whether the last point added to the path will cause a cable crossing and making the binary choice of over or under. Each time the robot crosses the cable a new visibility cell is created whose set of stitch lines contains the cable that the robot has recently crossed in addition to the set of stitch lines of its parent (see Fig. 7).

\section{B. Violation of the tree structure of MRLVAG}

For cable interactions, in addition to wrapping events, over and under events are needed, yielding the set $\{o, u, w\}$.

Theorem 3. With cable crossing events the configuration space no longer has genus 0 , i.e., the topological structure is changed so that the MRLVAG is no longer a tree.

Proof. Figure 7 provides an example by construction. It is possible to arrive at the same chart from two different charts, implying that there are loops in the atlas structure (in this case we can arrive at chart $\mathrm{C}$ either from chart $\mathrm{A}$ or $\mathrm{B}$ ).

The practical ramifications of this issue are resolved easily by finding any spanning tree of the MRLVAG. Since the MRLVA is itself complete, any of its spanning trees is complete as well. Nevertheless, the question remains: which spanning tree do we prefer? To answer this, we consider the effect of the topology on the optimality of the robot's movement. The order in which the events occur is related to how suboptimal the path taken by the robot can be.

Reexamining Fig. 7, we see that if the goal is reaching chart $\mathrm{C}$, then Fig. $7 \mathrm{~b}$ is a shorter distance than Fig. 7a. In fact, if the movement to cross the cable is arbitrarily small, then a robot going from chart $\mathrm{B}$ to chart $\mathrm{C}$ moves the same distance as if cable crossing were ignored entirely.

\section{Maintaining the Preferred Tree}

With a preferable atlas tree, we must generate that tree. If the robot knew which events were going to occur along a trajectory to its destination, the shortest route would start by connecting the current location of the robot to the first event, the first event to the second event, and so on until it connects the last event location to the destination. This 


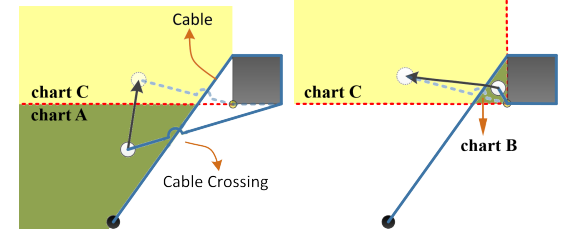

(a) from chart $\mathrm{A}$ to $\mathrm{C}$ (b) from chart $\mathrm{B}$ to $\mathrm{C}$

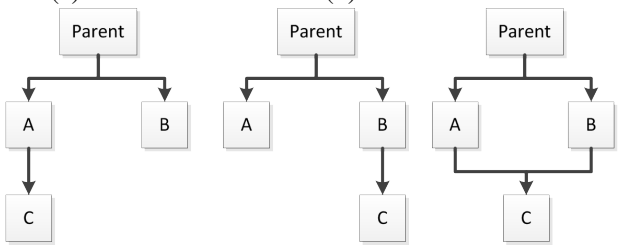

(c) From left to right: the tree made by movement in Fig. 7a, in Fig. 7b, and the inherent loop.

Fig. 7: An example of an MRLVAG growing toward a destination.

event ordering is the order in terms of their location on the cable (see Fig. 8). Thus, the algorithm is extended to swap events that are out of order. In Fig. 7a, for example, although the cable event happens before the wrapping event, since the position of the wrapping event is before the cable event on the cable, the algorithm will reorder them by replacing the wrapping event behind the cable events.

\section{Sequential Cable Events}

The precise configurations that result from cable-cable interactions depend on the physical properties of the cable. For our theoretical treatment, we simplify these complexities by assuming that whenever the cable wraps around itself there is an imaginary pin that prevents the displacement of the wrapping point as the robot moves. This models a cable with infinite friction with itself. This allows one to define a chart wherever the robot crosses a cable followed by crossing the same cable except with the other type of action (i.e., $o$ followed by $u$ or vice versa, see Fig. 9). The Base of this chart is the imaginary pin point and the cable is the stitch line. Such charts need not be stored permanently in the atlas tree, as there can be infinitely many different charts on a single cable (each depending on the location of the imaginary pin). A chart that is created because of a sequence of cable crossing events and all its children will be removed from the atlas whenever the robot's configuration leaves that chart. This illustrates the power of dynamically generating parts of the configuration space online.

\section{EXPERIMENTAL RESULTS}

To demonstrate the method and evaluate its performance, we developed a simulation environment and implemented the algorithm in C\# (see Fig. 10).

Fundamental differences between our method and existing work make picking appropriate criteria for comparison challenging. In particular, running time is problematic because other state-of-the-art methods employ a discretization of the configuration space. The running time is directly affected by the discretization resolution, and memory utilization suffers from the same illegitimacy. After careful consideration, it

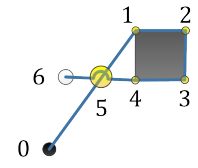

Fig. 8: The best ordering of events for reaching from the base point to the destination point. In other words, for having the shortest path, the points with smaller number should be reached earlier.
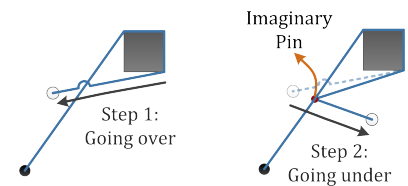

Fig. 9: An example of sequential cable crossing events.

was determined that the most equitable means for evaluation was to give a measure of the proportion of configuration space expanded by the algorithm, and the number of cells (i.e., an indication of memory footprint) used in doing so.

For each test scenario we calculated the total volume of the configuration space. We then compared it to the summed volume of the cells that are sufficient for specifying the robot and cable's configuration: current visibility cell and its parents up to the root. The results in Table 1 were generated by examining all points of the configuration space for the two test scenarios, and looking at the proportion of the configuration space volume stored in memory. The minimum and maximum rows report values for the cheapest and most costly to represent, respectively.

\begin{tabular}{|c||c|c||c|c|}
\hline \multicolumn{1}{|c||}{} & \multicolumn{2}{c||}{ Environment 1} & \multicolumn{2}{c|}{ Environment 2} \\
\hline $\begin{array}{c}\text { Memory } \\
\text { Usage }\end{array}$ & $\begin{array}{c}\text { \% of MRLVA } \\
\text { volume covered }\end{array}$ & $\begin{array}{c}\text { Cell } \\
\text { Count }\end{array}$ & $\begin{array}{c}\text { \% of MRLVA } \\
\text { volume covered }\end{array}$ & $\begin{array}{c}\text { Cell } \\
\text { Count }\end{array}$ \\
\hline Min & 62.52 & 1 of 36 & 7.12 & 1 of 115 \\
Max & 74.8 & 9 of 36 & 21.47 & 13 of 115 \\
\hline
\end{tabular}

TABLE 1: Results of the Experiments

The cable induces different degrees of topological complexity in environments with differing numbers and complexity of obstacles. To demonstrate this effect, we have chosen the two test scenarios in Fig. 11.

\section{A. On-line generation versus off-line generation}

Table 1 demonstrates the savings enabled by on-line generation of the configuration space. They show that if we were going to use a discretization of the configuration space represented as an MRLVA, storing the current chart and its ancestors would reduce the volume, the number of vertices in the graph, and consequently the memory use and searching time needed over the off-line methods used in the state-ofthe-art.

\section{B. Cell Decomposition Technique}

Although using the on-line atlas-based method reduces the number graph nodes, it is not the only advantage of the method. As explained in Section III-B, when the atlas is comprised of charts encoding the visibility properties of the environment, a special data structure can be used to represent the chart as a continuous space. Doing so requires only a 


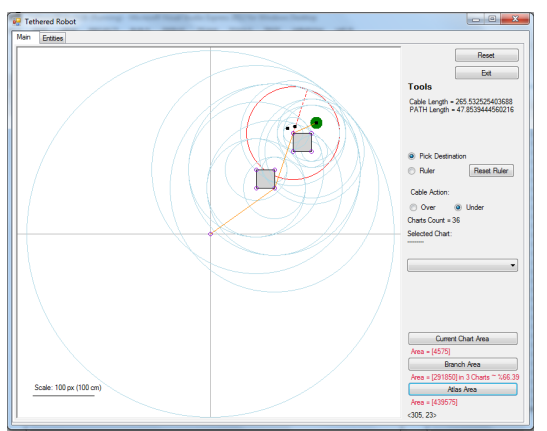

Fig. 10: Screen-shot of the simulation environment.

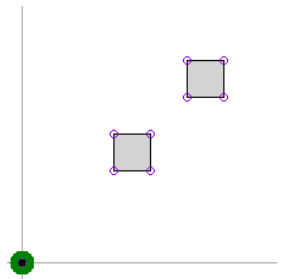

(a) Test Environment 1 (simple) (b) Test Environment 2 (complex)
Fig. 11: The two test environments used for presenting the results. Fig. 11a and Fig. $11 \mathrm{~b}$ are representative of simple and complex environments, respectively. Gray is obstacle and green is robot.

constant amount of memory no matter how big or small (in volume) the chart is.

Therefore, the number of charts used is a more important factor than area. We computed the number of nodes in the MRLVAG generated by the on-line method. The total number of charts needed for the configuration space depends on the complexity of the environment as it affects the manifold; consequently these numbers are reported too. Results for the test scenarios, shown in Table 1, illustrate that savings are substantial in both simple and complex manifolds.

\section{Cable Induced Manifold Structure}

The presented data also lead to an additional observation about memory saving in environments with different degrees of topological complexity. At one end of the scale, simple environments result in a configuration space that is mostly planar and has a small MRLVAG. In these cases, large volumes of the configuration space are captured with single cells. The environment in test scenario 1 is an exemplar: more than $60 \%$ of the configuration space is represented by a single chart. At the other end of the scale, complicated environments increase the topological complexity (reflected in large MRLVAGs) and have many visibility cells and charts. The resulting graphs are large, but have the form of wide and short trees. The environment in test scenario 2 illustrates this, with a total of 115 cells, but at most 13 ever need to be kept in memory. Thus, the cell decomposition approach results in significant memory saving across environment types.

\section{CONCLUSION}

This paper approaches planning for tethered robots with a new perspective: previous work employs a discretization of the configuration space along with an efficient search method. In addition to the need to perform substantial offline precomputation, existing approaches are unable to represent cable-cable interactions, and either ignore this problem or avert configurations which lead to them. The proposed method solves the basic tethered robot planning problem in a time and memory efficient way. Moreover, it is sufficiently general to form a consolidated representation for several other problems of interest, for example, winding constraints, some knot-like tying motions, etc. The method is, thus, more convenient and powerful than available approaches.

In this paper assumptions about the physical properties of the cable (tautness and friction) allow for well-defined cablecable interactions. Our immediate future work is incorporating the MRLVA while connecting multiple robots together with a cable. Another powerful property of the charts is their independence. One may use this in selecting distinct coordinate system representations for each chart to further improve efficiency. We are also interested in generalizing this concept to frictionless modeling of a cable as well.

\section{ACKNOWLEDGMENT}

This work was supported in part by the National Science Foundation as part of Grant IIS-1302393.

\section{REFERENCES}

[1] S. Lavalle, Planning algorithms. Cambridge University Press, 2006.

[2] S. Bhattacharya, V. Kumar, and M. Likhachev, "Search-based path planning with homotopy class constraints," in Annual Symposium on Combinatorial Search, 2010.

[3] T. Igarashi and M. Stilman, "Homotopic path planning on manifolds for cabled mobile robots," in Algorithmic Foundations of Robotics IX. Springer, 2011, pp. 1-18.

[4] D. S. Yershov, P. Vernaza, and S. M. LaValle, "Continuous planning with winding constraints using optimal heuristic-driven front propagation," in IEEE International Conference on Robotics and Automation (ICRA). IEEE, 2013, pp. 5551-5556.

[5] I. Shnaps and E. Rimon, "Online coverage by a tethered autonomous mobile robot in planar unknown environments," in Proceedings of Robotics: Science and Systems, Berlin, Germany, June 2013.

[6] J. M. Lee, Introduction to smooth manifolds. Springer, 2012, vol. 218.

[7] S. Hert and V. Lumelsky, "The ties that bind: Motion planning for multiple tethered robots," Robotics and autonomous systems, vol. 17, no. 3, pp. 187-215, 1996.

[8] D. Grigoriev and A. Slissenko, "Polytime algorithm for the shortest path in a homotopy class amidst semi-algebraic obstacles in the plane," in Proceedings of the 1998 international symposium on Symbolic and algebraic computation. ACM, 1998, pp. 17-24.

[9] V. Narayanan, P. Vernaza, M. Likhachev, and S. M. LaValle, "Planning under topological constraints using beam-graphs," in IEEE International Conference on Robotics and Automation (ICRA). IEEE, 2013 pp. 431-437.

[10] T. Lozano-Pérez and M. A. Wesley, "An algorithm for planning collision-free paths among polyhedral obstacles," Communications of the ACM, vol. 22, no. 10, pp. 560-570, 1979.

[11] T. Lozano-Pérez, "Spatial planning: A configuration space approach," Computers, IEEE Transactions on, vol. 100, no. 2, pp. 108-120, 1983.

[12] T. Krick, A. Slisenko, P. Solernó, and J. Heintz, "Search for shortest path around semialgebraic obstacles in the plane," Journal of Mathematical Sciences, vol. 70, no. 4, pp. 1944-1949, 1994.

[13] M. de Berg, M. van Kreveld, M. Overmars, and O. Schwarzkopf, Computational Geometry, Algorithms and Applications. Springer, 1991.

[14] R. H. Teshnizi and D. A. Shell, "Computing cell-based decompositions dynamically for planning motions of tethered robots," http:// engineering.tamu.edu/media/1136933/2014-2-3.pdf, CSE Dept., Texas A\&M Univ., Tech. Rep. 2014-2-3, 2014. 